\title{
Robot-assisted laparoscopic radical cystectomy: initial experience on 27 consecutive patients
}

\author{
A. Mottrie $\cdot$ P. Carpentier $\cdot$ P. Schatteman $\cdot$ E. Fonteyne $\cdot$ \\ H. Suttmann $\cdot$ M. Stöckle $\cdot$ S. Siemer
}

Received: 14 June 2007 / Accepted: 26 July 2007 / Published online: 15 August 2007

(C) Springer London 2007

\begin{abstract}
Recent reports have demonstrated that robotassisted laparoscopic cystectomy is technically feasible. We report technical and functional results of a large series of patients undergoing laparoscopic cystectomy with the da Vinci surgical system (DVSS). A total of 27 patients (24 males) underwent laparoscopic radical cystectomy with the DVSS (intuitive surgical) between January 2004 and December 2005. Indications for cystectomy were muscleinvasive transitional cell carcinoma (TCC) or leiomyosarcoma of the urinary bladder $(n=24)$ and bladder shrinking following prior radiotherapy for TCC. A pelvic lymphadenectomy was a routine part of the procedure. Urinary diversions were ilieal conduits $(n=19)$ and ileal neobladders $(n=8)$. Mean operating time was $340 \mathrm{~min}$ (range 150-450) with a mean blood loss of $301 \mathrm{ml}$ (range 50-550). The mean number of lymph nodes retrieved during lymphadenectomy was 23. Surgical margins were negative except in one case. After a mean follow-up of 10.2 months, two perioperative (anastomotic leakage, adhesions) and three postoperative complications (ileus, intestinal fistula, urinary tract obstruction) occurred. Six out of seven patients reported satisfying erectile function following nerve-sparing surgery. Day-time continence was completely restored
\end{abstract}

\footnotetext{
A. Mottrie - P. Carpentier · P. Schatteman - E. Fonteyne Department of Urology, Onze-Lieve-Vrouw Ziekenhuis, Aalst, Belgium

e-mail: a.mottrie@telenet.be

H. Suttmann · M. Stöckle $\cdot$ S. Siemer $(\square)$ Department of Urology and Pediatric Urology,

Saarland University Hospital, Kirrbergerstrasse,

66421 Homburg/Saar, Germany

e-mail: urssie@uniklinikum-saarland.de
}

after a mean 3.5 months in seven of eight patients. Robotassisted laparoscopic cystectomy is a safe procedure. Satisfying functional and oncological short-term results can be achieved within acceptable operating time limits.

Keywords Robot · Da Vinci · Laparoscopy · Bladder cancer · Cystectomy · Urinary diversion

\section{Introduction}

Minimally invasive surgery has revolutionized most fields of medicine. In urology, the era began with laparoscopy for undescended testes and subsequent orchidopexy in children. Today, laparoscopy encompasses the management of many benign and malignant urologic disorders, and laparoscopic nephrectomies are a standard procedure in many centers [1]. Even for challenging urological tumor surgery such as radical prostatectomy, the advantages of minimally invasive surgery are a faster time to recovery from surgery, shorter off-work periods and reduced pain. In contrast, laparoscopic urologic surgery has certain drawbacks including limited instrumentations, a lack of training cases, a steep learning curve and reconstructive techniques that are difficult to execute. Further controversy exists regarding functional and oncological long-term outcome, especially in tumor surgery [1-3].

Robotic assistance significantly reduces the technical challenge and learning curve and extends the potential for an "open surgeon" to embrace these laparoscopic developments more easily [3-10]. The da Vinci system has 3D vision, seven degrees of freedom of movement and an articulating robotic EndoWrists ${ }^{\circledR}$ (Intuitive Surcical, Sunnyvale, CA) that mimic the surgeon's hand movements. This expansion of traditional laparoscopy facilitates a more 
precise tissue handling, which is especially important in performing technically challenging procedures such as preservation of neurovascular bundles or urinary diversion. Thus, the introduction of robot-assisted laparoscopy offers the inherent advantages of minimally invasive surgery while improving the surgeon's learning curve and ability to perform difficult operations [3-10].

In the present study we report on the advantages and disadvantages of robot-assisted laparoscopic radical cystectomy with urinary diversion using the DVSS in a large cohort of patients with malignant disorders of the urinary bladder.

\section{Patients and methods}

\section{Background and patient selection}

Laparoscopic surgery has been performed in our departments since 1997, and in 2002 the DVSS was introduced. After establishing robot-assisted radical prostatectomy in 290 patients over 3.5 years, we started gaining experience with robot-assisted cystectomy. A total of 27 patients (24 males, 3 females) with a history of muscle-invasive TCC or leiomyosarcoma of the urinary bladder underwent robotassisted laparoscopic radical cystectomy between January 2004 and December 2005. Preoperative diagnostic work-up included abdominal ultrasound, CT scans and chest X-rays. Patients had to have clinically organ-confined disease without gross enlargement of lymph nodes to be eligible for robot-assisted surgery. The decision for the kind of urinary diversion was based on critically weighing various parameters such as patient age, gender and preference as well as tumor stage. A pelvic lymphadenectomy was performed as a routine part of the procedure.

\section{Surgical technique}

We used a three-arm DVSS to perform laparoscopic radical cystectomy. Bowel preparation was initiated 1 day before the procedure. On the morning of surgery a broad-spectrum antibiotic was administered, and bilateral leg compression stockings were applied. Under general anesthesia a nasogastric tube and a foley transurethral catheter were inserted. Patients were placed in extended lithotomy position with both legs tightly secured in boot holders, hips lightly flexed and knees at about $60^{\circ}$. Trendelenburg positioning of approximately $25^{\circ}$ was applied to allow the bowels to fall back and open the pelvic cavity.

After insufflation of the peritoneum with carbon dioxide using a Veress needle up to an intra-abdominal pressure of $12 \mathrm{mmHg}$, a total of six ports were inserted. The first port $(12 \mathrm{~mm})$ was placed $3 \mathrm{~cm}$ above the umbilicus and a $0^{\circ}$-laparoscope was inserted for an initial inspection of the peritoneal cavity. The other ports (two 8-mm ports for robot arms, a $12-\mathrm{mm}$ port and two $5-\mathrm{mm}$ ports for assistance at the operating table) were placed under direct vision. In male patients, the Douglas space was opened to expose the dorsal aspect of the vasa deferentia and seminal vesicles. Denonvillier's fascia was incised to gain access to the space between the prostate and rectum. The dorsal side of the prostate was mobilized down to the apex. In female patients, the peritoneum was incised at the level of the cervix or upper rim of the vagina if a hysterectomy had been performed previously. In case of a present uterus, en-block resection of the uterus together with the cystectomy specimen was performed.

Afterwards, both ureters were identified and mobilized down to the vesicoureteral junction respecting their vasculature. Two clips each were placed distally and the ureters cut in between. Distal resection margins were sent to pathology for frozen section analysis. The ureters remained clipped until performing urinary diversion to allow for dilation and an easier suture of the subsequent anastomosis.

Following this step, a pelvic lymphadenectomy was performed. The retroperitoneal and iliac vessels were exposed, and lymphatic tissue was removed using the split-and-roll technique starting at about $3 \mathrm{~cm}$ above the aortic bifurcation. Lymphatic tissue in the upper and lower obturator fossa was mobilized and left on the bladder specimen for en-block resection. Cystectomy was mainly performed as described by Menon et al. [11, 12]. The proximal urethra was closed by a circular suture and transected distally to avoid urine spillage. The specimen was entrapped and retrieved in an organ bag. Afterwards, bowel around the cecum was mobilized.

For ileal conduits, the left ureter was swung to the right side over the sacral promontory behind the sigmoid mesocolon. Urinary diversion was performed using an extracorporeal technique through a $4-5 \mathrm{~cm}$ minilaparotomy. For ileal neobladders, a W-pouch with double chimney was used. The ureters were left in place and an end-to-end anastomosis between the ureter and chimney was performed at both sides above the crossing of the common iliac vessels.

\section{Results}

Patient characteristics and details regarding the surgical technique are summarized in Tables 1, 2. Mean total operating time as determined from inserting the veress needle to definite skin closure was 340 min (range 150-450). Mean blood loss was $301 \mathrm{ml}$ (range 50-550). Postoperative blood transfusion was necessary in $2 / 27$ patients. Eight patients received an orthotopic ileal neobladder. In seven male patients, nerve-sparing cystectomy was performed. 
Table 1 Patient characteristics

\begin{tabular}{ll}
\hline Total & $n=27$ \\
\hline Age & 63 years (range 40-82) \\
Mean ASA score & 2.6 \\
Gender & Male $n=24$ \\
& Female $n=3$ \\
Preoperative histology & \\
TCC $\geq \mathrm{T}_{1} \mathrm{G}_{\mathrm{III}}$ & $n=23$ \\
Leiomyosarcoma & $n=1$ \\
Shrinking bladder following radiotherapy & $n=3$ \\
\hline
\end{tabular}

Table 2 Surgical technique and complications

\begin{tabular}{ll}
\hline Urinary diversion & \\
\hline Ileal conduit & $n=19$ \\
Ilial neobladder & $n=8$ \\
Synchronous urethrectomy & $n=6$ \\
Mean follow-up & 10.2 months (range 1-25) \\
Complications & \\
Perioperatively & $n=2$ \\
& (Anastomotic leakage, adhesions) \\
Postoperativly & $n=3$ \\
& (Ileus, intestinal fistula) \\
Upper urinary tract obstruction & $n=1$ \\
Nerve-sparing & $n=7$ \\
With satisfactory & $n=6$ \\
postoperative potency & \\
\hline
\end{tabular}

Details on uro-oncological outcome are listed in Table 3. Three shrinking bladders after radiotherapy were tumor free. Six patients had non-organ confined disease (stages pT3a, pT3b or pT4). Surgical margins were negative except in one patient. In this patient with negative results on frozen section analysis, the final histopathology workup revealed carcinoma in situ at the distal ureteral margins of the left ureter. The mean number of lymph nodes retrieved on lymphadenectomy was 23 (range 6-37). Three of these patients had histologically proven nodal involvement $(\mathrm{pN}+)$.

Complications are listed in Table 2. At a mean follow-up of 10.2 months, two perioperative and three postoperative complications occured in our cohort. In one patient, a postoperative volvulus caused obstructive ileus, and $20 \mathrm{~cm}$ of small bowel had to be resected, subsequently. The initial enteroanastomosis was intact. In one patient we note an ureterointestinal anastomotic stricture after ileal conduit diversion. Six out of seven male patients reported on satisfactory erectile function with successful intercourse after nerve-sparing cystectomy, two of them are on intermittent PDE-5 inhibitors.
Table 3 Postoperative histopathologic results

\begin{tabular}{ll}
\hline & $n(\%)$ \\
\hline No residual tumor & $3(11)$ \\
Malignant & $24(89)$ \\
Tumor stage & \\
pT1 & $3(13)$ \\
pT2a & $7(29)$ \\
pT2b & $8(33)$ \\
pT3a & $1(4)$ \\
pT3b & $2(8)$ \\
pT4 & $3(13)$ \\
Tumor grade & \\
GI & 0 \\
GII & $5(21)$ \\
GIII & $19(79)$ \\
Nodal status & \\
pN0 & $21(91)$ \\
pN+ & $4(9)$ \\
Surgical margins & \\
R0 & $23(96)$ \\
R1 & $1(4)$ \\
R2 & 0 \\
\hline
\end{tabular}

Postoperative continence in the patients with ileal neobladder is excellent. Day-time continence was fully restored after a mean follow-up of 3.5 months in seven patients. In one patient, two pads per day are needed after a follow-up of 6 months.

\section{Discussion}

Since the early 1980s, minimally invasive surgery in urology has been constantly improving through technical, instrumental and surgical advances. Today, the advantages of laparoscopic procedures as compared to classic open surgery are widely acknowledged and include improvements in surgery-associated pain, better cosmetic results and faster convalescence from surgery. Even oncologic outcome appears to be comparable between minimally invasive and open surgical techniques [1, 7, 13].

Radical cystectomy with urinary diversion is the standard treatment for muscle-invasive TCC of the urinary bladder. It took almost 12 years from the dawn of laparoscopic urology before the first attempts of performing laparoscopic radical cystectomy were published. Parra et al. [14] and Sanchez de Badajoz et al. 15] reported on their initial experience with laparoscopic cystectomy with extracorporeal urinary diversion in 1992 and 1993, respectively. Additional case reports followed in the mid-1990s [16]. The first larger series with $n=9$ (Abdel-Hakim et al. [18]), 
$n=10$ (Hemal et al. [17]), and $n=14$ (Menon et al. [11]) patients were presented at the beginning of the twenty-first century. Gill et al. [19] published results on laparoscopic cystectomy with intracorporeal suture of the enteroanastomosis and complete urinary diversion in 2000. Using the DVSS, this procedure was performed for the first time with a robot-assisted approach by Balaji et al. [20] at the University of Nebraska. The operating times of Balaji et al. [20] in three patients were 616, 626 and $828 \mathrm{~min}$, respectively.

In our series, urinary diversion was performed via the extracorporeal technique. Even when using the DVSS to assist in reconstructive surgery, prolonged duration of surgery of about $3 \mathrm{~h}$ has to be calculated when comparing extracorporeal and intracorporeal techniques. In the report by Balaji et al. [20], an about 5-cm minilaparotomy incision was necessary to recover the bladder specimen. We used a similar incision to place the relevant intestinal segments extracorporeally and perform the urinary diversion as well as the enteroanastomosis. For ilieal neobladders, anastomotic sutures were put in place at the urethral stump before undocking the DVSS and tying the urethroneovesicostomy. This technique lead to a reduction in the total operating time.

3D vision and the articulating EndoWrist of the DVSS allow for very subtle tissue handling and fine dissections, which resulted in an acceptable mean operating time of $340 \mathrm{~min}$ in our study. Functional and oncological outcome in our patient cohort was excellent. Only one patient had positive surgical margins with carcinoma in situ at the distal left ureter following negative histopathological results on frozen section analysis. Obviously, this finding cannot be attributed to surgical technique. The average blood loss was $301 \mathrm{ml}$, which compares favorably with the results in other series [3-11, 17-20]. Only two patients with a low preoperative hemoglobin had to have blood transfused. Results of the nerve-sparing technique are promising, yet still preliminary and hampered by the low number of patients in our study. However, we feel that the advantages of using the DVSS become evident, expecially when performing preservation of the neurovascular bundles.

Radical open tumor surgery is being more and more replaced by minimally invasive surgery, a trend that is not only evident in urology. At the beginning of the laparoscopy era, long operating times and high costs were put forward as an argument against these new surgical techniques by their opponents. However, the advantages of laparoscopic surgery with excellent postoperative results will promote continuing to implement these procedures as an integral component of providing medical care to our patients. From our point of view, robot-assisted laparoscopy with the DVSS does not compete with conventional laparoscopy. It rather represents a further refinement that makes it easier for novices in this field to gain experience in minimally invasive surgery. Thus, laparoscopic surgery becomes an option to consider even for centers with little or no prior experience. It has to be assumed that the initial high costs for establishing robot-assisted surgery will continue to decrease within the next years.

\section{Conclusions}

Robot-assisted laparoscopic radical cystectomy is a safe and effective procedure that can be performed within acceptable operating time limits. It provides excellent functional and oncological results with low complication rates. The additional value of performing an intracorporeal urethroneovesicostomy and urinary diversion remains controversial.

\section{References}

1. Gill IS, Kerbe K, Meraney AM (2002) Basics of laparoscopic urologic surgery. In: Walsh PC, Retik AB, Vaughan ED, Wein AJ (eds) Campbell's urology. Saunders, Philadelphia, pp 3455-3505

2. Rassweiler J, Schulze M, Teber D, Seemann O, Frede T (2004) Laparoscopic radical prostatectomy: functional and oncological outcomes. Curr Opin Urol 14:75-82

3. Hemal AK, Menon M (2004) Robotics in urology. Curr Opin Urol 14:89-93

4. Gettman MT, Blute ML, Peschel R, Bartsch G (2003) Current status of robotics in urologic laparoscopy. Eur Urol 43:106-112

5. Kaul S, Shah NL, Menon M (2006) Learning curve using robotic surgery. Curr Urol Rep 7:125-129

6. Binder J, Bräutigam R, Jonas D, Bentas W (2004) Robotic surgery in urology: fact or fantasy? BJU Int 94:1183-1187

7. Hemal AK, Abol-Enein H, Tewari A et al. (2004) Robotic radical cystectomy and urinary diversion in the management of bladder cancer. Urol Clin North Am 31:719-729

8. Rimington P, Dasgupta P (2004) Laparoscopic and robotic radical cystectomy. BJU Int 93:460-461

9. Miller NL, Theodorescu D (2006) Status of robotic cystectomy in 2005. World J Urol 24:180-187

10. Shah NL, Hemal AK, Menon M (2005) Robot-assisted radical cystectomy and urinary diversion. Curr Urol Rep 6:122-125

11. Menon M, Hemal AK, Tewari A et al. (2003) Nerve-sparing robot-assisted radical cystoprostatectomy and urinary diversion. BJU Int 92:232-236

12. Menon M, Hemal AK, Tewari A et al. (2004) Robot-assisted radical cystectomy and urinary diversion in female patients: technique with preservation of the uterus and vagina. J Am Coll Surg 198:386-393

13. Jackson CL (2001) Urologic laparoscopy. Surg Oncol Clin N Am 10:571-578

14. Parra RO, Andrus CH, Jones JP, Boullier JA (1992) Laparoscopic cystectomy: initial report on a new treatment for the retained bladder. J Urol 148:1140-1144

15. Sanchez de Badajoz E, Gallego Perales JL, Reche Rosado A, Gutierrez de la Cruz JM, Jimenez Garrido A (1995) Laparoscopic cystectomy and ileal conduit: case report. J Endourol 9:59-62

16. Hubert J, Chammas M, Larre S et al. (2006) Initial experience with successful totally robotic laparoscopic cystoprostatectomy and 
ileal conduit construction in tetraplegic patients: report of two cases. J Endourol 20:139-143

17. Hemal AK, Singh I, Kumar R (2003) Laparoscopic radical cystectomy and ileal conduit reconstruction: preliminary experience. J Endourol 17:911-916

18. Abdel-Hakim AM, Bassiouny F, Abdel Azim MS et al. (2002) Laparoscopic radical cystectomy with orthotopic neobladder. J Endourol 16:377-381
19. Gill IS, Fergany A, Klein EA et al. (2000) Laparoscopic radical cystoprostatectomy with ileal conduit performed completely intracorporeally: the initial 2 cases. Urology 56:26-30

20. Balaji KC, Yohannes P, McBride CL, Oleynikov D, Hemstreet GP3 (2004) Feasibility of robot-assisted totally intracorporeal laparoscopic ileal conduit urinary diversion: initial results of a single institutional pilot study. Urology 63:51-55 